\title{
Validation of an Instrument to Control and Monitor the Training Load in Basketball: The BATLOC Tool
}

\author{
D. Berdejo-del-Fresno ${ }^{1,2, *}$, J.M. González-Ravé ${ }^{3}$ \\ ${ }^{1}$ The Football Association, London, England \\ ${ }^{2}$ Sheffield FC Futsal, England \\ ${ }^{3}$ Sport Training La, Faculty of Sport Sciences, University of Castilla-La Mancha, Spain \\ *Corresponding author: daniberdejo@gmail.com \\ Received July 14, 2014; Revised August 01, 2014; Accepted August 05, 2014
}

\begin{abstract}
The main objective of a coach is to optimize athletic performance. The best performance improvements come from prescribing an optimal dose of physical training with proper recovery periods to allow for the greatest adaptation before competition. The main objective was to validate an inexpensive, easy, non-invasive, real time tool to control and monitor the training load in basketball: the BATLOC tool. Fourteen elite female basketball players from a top-4 team that competes in the England Basketball League Division I volunteered to participate in this study ( $20.50 \pm 2.31$ years old, $174.21 \pm 4.17 \mathrm{~cm}, 75.21 \pm 15.38 \mathrm{~kg}$, BMI of $24.67 \pm 4.23,177.29 \pm 7.60 \mathrm{~cm}$ of arm span, $19.01 \pm 2.34 \%$ of body fat, and $45.18 \pm 4.17 \mathrm{ml} / \mathrm{kg} / \mathrm{min}$ of VO2max. Two mesocycles were analized: pre-season (6 weeks) and in-season (10 weeks). Training load was controlled and monitored daily with the BATLOC tool. Heart rate was monitored for every player every $5 \mathrm{~s}$ in each training session. The RPE was measured using the 6-20 Borg scale. The Pearson's product moment correlation between the means of intensity, RPE, heart rate, maximum heart rate and equivalent training load showed an excellent concordance ( $>0.75)$. To conclude, based on the results in this study and the literature reviewed, the BATLOC tool seems to be a good method to control global internal training load in basketball. This method does not require any expensive equipment and may be very useful and convenient for coaches to monitor the internal training load of basketball players.
\end{abstract}

Keywords: RPE, heart rate, periodisation, team sports

Cite This Article: D. Berdejo-del-Fresno, and J.M. González-Ravé, "Validation of an Instrument to Control and Monitor the Training Load in Basketball: The BATLOC Tool." American Journal of Sports Science and Medicine, vol. 2, no. 4 (2014): 171-174. doi: 10.12691/ajssm-2-4-10.

\section{Introduction}

The main objective of a coach is to optimize athletic performance [1]. The best performance improvements come from prescribing an optimal dose of physical training with proper recovery periods to allow for the greatest adaptation before competition [1,2]. Physical training is the systematic repetition of physical exercises, and it can be described in terms of its outcome (anatomical, physiological, biochemical, and functional adaptations) or its process, that is, the training load [3]. The stimuli for training induced adaptations is the relative physiological stress imposed on the athletes (internal training load) and not the external training load (e.g. $10 \times 500 \mathrm{~m}$ at $3 \mathrm{~min} / \mathrm{km}$ ) [3]. Therefore, to monitor and control the training process, it is important to have a valid measure of internal training load [4]. This is particularly relevant in team sports where the planned external load is often similar for each team player because of the extensive use of group exercises such as small-sided games in team training sessions [5].

Many different methods of recording training loads in sports have been reported. Some of these methods have included measurement of heart rates [6], distance covered during training [7], weights lifted [8], repetitions completed, training time, or session-Rating of Perceived Exertion (RPE) $[9,10])$. Since heart rate seems to be one of the best objective ways to quantify aerobic training intensity [11,12], many of the methods to quantify the internal training load are based on heart rate monitoring $[6,13]$. However, the routine use of heart rate-based methods is not always feasible due to problems such as the required technical expertise, the time-consuming process of collecting heart rate data of all team players every training session, and most importantly the cost of numerous heart rate telemetric systems. Furthermore, one more problem with using heart rate methods is that the heart rate transmitter belts are not allowed during official competitive matches. This is an important limitation because the match training load may be a relative high percentage of the weekly training load.

An alternative strategy was developed by Foster [9] or Foster et al. [10]. The session-RPE method to monitor training load requires each athlete to provide a Rating of Perceived Exertion (RPE) for each exercise session along with a measure of training time $[9,10]$. The product of both values represents in a single number the magnitude of internal training load in arbitrary units (AU). This 
method has been significantly correlated to the HR-based method of quantifying internal training load proposed by Edwards [14], and has recently been applied to basketball $[4,15,16,17]$. Therefore, it can be stated that the Foster et al. $[9,10]$ method can be perfectly used to compare, correlate and confirm the potential validation of the BATLOC tool (BAsketball Training LOad Control Tool) [18], since it has been proved to be a valid, reliable and useful method to monitor and control training load in basketball.

Nevertheless, both previous methods are either too expensive (heart rate monitors) or not able to work in real time or until the training session has finished (session$\mathrm{RPE}$ ). These are the main reasons why in team sports the training load has generally been calculated using the RPE method or the TRIMP method [5,16,17,19,20,21]. This way, the training load is calculated once the training session has finished, avoiding the chance of receiving feedback on the training load in real time or the opportunity to modify the session in that moment.

Moreover, since all the quantification methods are imperfect by nature (and so is the present model), the main objective of this study was to validate an inexpensive, easy, non-invasive, real time tool to control and monitor the training load in basketball: the BATLOC tool [18]. It is a method that can be used for all teams, regardless of their gender, level or budget. For its validation, the training load obtained from the BATLOC tool will be correlated to the session-RPE, the heart rate and the method developed by Foster and colleagues $[9,10]$.

\section{Methods}

\subsection{Participants}

Fourteen elite female basketball players from a top-4 team that competes in the England Basketball League Division I volunteered to participate in this study after having signed the corresponding informed consent. The team was made up by 2 members of the Senior Great Britain Women Basketball Team, a member of the Under 20 Great Britain Women Basketball Team and Under 18 England Women Basketball Team, a member of the Under 20 Great Britain Women Basketball Team and Under 18 Scottish Women Basketball Team, 2 players of the Under 20 Hungary Women Basketball Team, 2 USA professional players, and 6 non-international British players. This study was approved by the local Ethics Committee and conducted in accordance with the guidelines of the revised Declaration of Helsinki.

\subsection{Anthropometric Tests}

Anthropometric measures were taken following the Lohmann et al. [22] instruction. Standing height and arm span were measured with a precision of $0.1 \mathrm{~cm}$ with a stadiometer and a tape measure, respectively (SECA Ltd, model 220, Germany). Body mass (kg) was recorded with a scale SECA (SECA Ltd, Germany) to the nearest $100 \mathrm{~g}$, the subjects wearing light, indoor clothing and no shoes. The Body Mass Index (BMI) was calculated using the Quetelet formula.

\subsection{Training Load (BATLOC Tool)}

Training load was controlled and monitored daily with the BATLOC tool [18]. The BATLOC tool is a software designed with Microsoft Office Excel to control and monitor the training load in real time in basketball sessions. The software uses a database with basketball exercises and their corresponding given training load value. The software allows the coach to add different and more exercises to the database just in case they are not included. The training load value pre-assigned to each exercise or drill is calculated taken into account 4 variables of each exercise: heart rate, density, opposition and distance during the development of the drill. For example, the exercise " $5 \times 52$ courts" obtained the following values: 8 points in the heart rate aspect, 9 in density, 10 in opposition or number of players involved, and 7 in distance (mean: 8.5 points). Thus, with a simple rule of three, this exercise showed a training load of 23.8 $[(28 * 8.5) / 10=23.8]$. This means that if any coach performs the exercise " $5 \times 52$ courts" for 10 minutes, the training load will be 23.8. If the exercise is practiced for 20 minutes, the training load will be 47.6. Therefore, during the session, one of the coaches must only control the duration (i.e. 10 minutes) of each exercise and introduce it together with the task's name (i.e. $5 \times 52$ courts) in the software (spreadsheet in Microsoft Office Excel). The software itself will calculate the training load for each drill and at the end of the spreadsheet the total training load of the session (total summation of the each drill's training load)[18]. Furthermore, the software will provide at the end of the sessions the following extra variables: session duration in minutes and equivalent training load. The equivalent training load is a classification of the session in relation to its total training load. The different sessions were 8 different types: tactical/shooting session refers to equivalent training load 0.5 (total training load < 50); technical 1/pre-game corresponds to equivalent training load 1 (total training load < 70); technical 1.5 goes with equivalent training load 1.5 (total training load < 90); technical 2 refers to equivalent training load 2 (total training load < 110); technical 2.5 corresponds to equivalent training load 2.5 (total training load < 130); technical 3 means equivalent training load 3 (total training load < 150); technical 3.5 goes with equivalent training load 3.5 (total training load $<170$ ); and technical 4/game means equivalent training load 4 (total training load $>170$ ) (Table 3). Therefore, a session with a total training load of 115.4 is considered as a technical 2.5 session or equivalent training load 2.5, since the total training load is $<130$.

Besides, the session's intensity was calculated with the equation: intensity = training load/duration.

Two mesocycles were analyzed: pre-season (6 weeks) and in-season (Competition I phase) (10 weeks). That research period covered the training load of a total of 50 tactical/technical sessions. Therefore, a total number of 700 individual training sessions were analysed (50 sessions $x 14$ players). If one player did not perform the whole session, the training load recorded was the load achieved until that moment.

\subsection{Heart Rate Control}

Heart rate was monitored for every player every $5 \mathrm{~s}$ in each training session using a heart rate monitor with individually coded transmitters via short-range 
radiotelemetry (Polar Team Sport System, Polar Electro, Finland). The variable used in this study was the mean heart rate for the whole practice. The data recorded during the briefing before the start of each training session were deleted. To reduce any heart rate recording errors during training, all players were regularly asked to check that their heart rate monitors were working and properly worn (at least every $10 \mathrm{~min}$ ). In addition to this, one of the researchers was permanently looking at the portable PC screen, making sure that every player's heart rate monitor was transmitting the data. After every training session, the heart rate data were exported and analysed using the Excel software programme (Microsoft Corporation, U.S.). The research period covered the heart rate of a total of 700 individual tactical/technical sessions. If one player did not perform the whole session, the heart rate recorded was the average rate achieved until that moment.

\subsection{Rating of Perceived Exertion}

The RPE was measured using the 6-20 Borg scale [23] (Table 1). Each player's session-RPE was collected about 30 min after each training session to ensure that the perceived effort was referring to the whole session rather than the most recent exercise intensity. All players were taught and familiarized with this scale for rating perceived exertion during the 2 weeks prior to the start of the study. In the procedure, the player is shown the scale and asked "How was your workout?", and they must give a single number representing the training session. The research period covered the session-RPE of a total of 700 individual tactical/technical sessions. If one player did not perform the whole session, the RPE recorded was the number given at the moment when the player withdrew from the session.

Table 1. Borg's 6-20 scale that is to be shown to the players $30 \mathrm{~min}$ after every training session

\begin{tabular}{cc} 
after every training session & \\
\hline Rating & Descriptor \\
\hline 6 & No exertion at all \\
7 & Extremely light \\
8 & Very light \\
10 & \\
11 & Light \\
12 & \\
13 & Somewhat hard \\
14 & \\
15 & Hard (heavy) \\
16 & \\
17 & Very hard \\
18 & \\
19 & Extremely hard \\
20 & Maximal exertion \\
\hline
\end{tabular}

\subsection{Statistical Analyses}

All data are presented as mean \pm standard deviation (s). The relationships between the session-RPE and the heart rate with the various variables given by the BATLOC tool were analysed using Pearson's product moment correlation. Fleiss' [24] evaluation defines concordance of variables as excellent when the correlation coefficient is $>0.75$, good when it is $0.60-0.74$, acceptable when 0.40 .0 .59 , and poor when $<0.40$. In the present study there were 5 variables with an excellent correlation (sessionRPE with intensity, training load and equivalent training load; and heart rate with training load and equivalent training load) and one variable with a good correlation (heart rate with intensity). There were no variables with a poor correlation.

\section{Results}

The players' physical and anthropometrical characteristics were as follows (mean \pm s): an age of 20.50 \pm 2.31 years old, a height of $174.21 \pm 4.17 \mathrm{~cm}$, a mass of $75.21 \pm 15.38 \mathrm{~kg}$, a Body Mass Index (BMI) of $24.67 \pm$ 4.23, an arm span of $177.29 \pm 7.60 \mathrm{~cm}$, a \% body fat of $19.01 \pm 2.34$, and an indirect VO2max of $45.18 \pm 4.17$ $\mathrm{ml} / \mathrm{kg} / \mathrm{min}$, calculated from the 20 -meter shuttle run test.

The distribution of the analysed technical/tactical session organised by their type is presented in Table 3, which also includes mean $\pm \mathrm{s}$ of session duration, training load, intensity, heart rate and heart rate max obtained from every type of training session. The Pearson's product moment correlation between the means of intensity, RPE, heart rate, maximum heart rate and equivalent training load showed an excellent concordance ( $>0.75$ ). Practices averaged $88.59 \pm 22.04 \mathrm{~min}$.

Session-RPE, heart rate, and heart rate max correlation with the variables given by the BATLOC tool for the 700 individual training sessions are shown in Table 2. The session-RPE had an excellent correlation with intensity $(\mathrm{r}=0.90)$, training load $(\mathrm{r}=0.80)$ and equivalent training load $(\mathrm{r}=0.76)$. Heart rate obtained two excellent correlations with training load $(r=0.87)$ and equivalent training load ( $\mathrm{r}=0.78)$, and one good correlation with the session intensity $(r=0.69)$. In addition, the maximum heart rate recorded during the session was correlated with the BATLOC's variables. However, these correlations were not as significant as the previous correlations.

Table 2. Pearson's product moment correlations (total data analysed $=700$ )

\begin{tabular}{cccc}
\hline & Intensity & Training Load & $\begin{array}{c}\text { Equivalent } \\
\text { Training Load }\end{array}$ \\
\hline Session-RPE & 0.90 & 0.80 & 0.76 \\
$\begin{array}{c}\text { Heart Rate } \\
\text { Maximum Heart } \\
\text { Rate }\end{array}$ & 0.69 & 0.87 & 0.78 \\
\hline
\end{tabular}

Finally, the correlation between the session-RPE and the average heart rate of the sessions was also calculated, even if it was not the main goal of this study ( $\mathrm{r}=0.92)$.

\section{Discussion}

The purpose of this research was to investigate the potential correlation and therefore validate an inexpensive, easy, non-invasive, real time tool to control and monitor training load in basketball: the BATLOC tool. More specifically, the correlations between the training load obtained from the BATLOC tool and the players' sessionRPE and heart rate were analysed with the aim of validating the new method. The present study is the first to apply the BATLOC tool and the players' session-RPE and heart rate. The correlations found (ranging from 0.69 to 0.90), classified as excellent and good [24], confirmed that 
the BATLOC tool may be an adequate and useful method

to control and monitor training load in basketball.

Table 3. Type of sessions analysed (total data analysed $=700)($ mean $\pm \mathrm{s})$

\begin{tabular}{|c|c|c|c|c|c|c|c|c|c|}
\hline \multicolumn{3}{|c|}{ Training Session Characteristics } & \multicolumn{7}{|c|}{ Analysed Training Session (mean $\pm s$ ) } \\
\hline $\begin{array}{l}\text { Session } \\
\text { Type }\end{array}$ & $\begin{array}{c}\text { Equivalent } \\
\text { Training } \\
\text { Load }\end{array}$ & $\begin{array}{c}\text { Training } \\
\text { Load } \\
\text { Range } \\
\end{array}$ & $\mathbf{n}$ & $\begin{array}{c}\text { Session } \\
\text { Duration (m) }\end{array}$ & $\begin{array}{c}\text { Training } \\
\text { Load }\end{array}$ & Intensity & RPE & $\begin{array}{l}\text { Heart Rate } \\
\text { (bpm) }\end{array}$ & $\begin{array}{l}\text { Heart Rate } \\
\text { max (bpm) }\end{array}$ \\
\hline $\begin{array}{l}\text { Tactical/ } \\
\text { Shot } 0.5\end{array}$ & 0.5 & $0-49$ & 28 & $44.00 \pm 1.41$ & $19.00 \pm 7.21$ & $0.43 \pm 0.18$ & $7.05 \pm 0.07$ & $89.50 \pm 0.71$ & $141.00 \pm 8.49$ \\
\hline $\begin{array}{l}\text { Technical } 1 \\
\text { (pre-game) }\end{array}$ & 1 & $50-69$ & 70 & $61.00 \pm 10.12$ & $52.03 \pm 2.52$ & $0.88 \pm 0.21$ & $10.25 \pm 0.50$ & $100.50 \pm 0.71$ & $154.00 \pm 0.71$ \\
\hline $\begin{array}{c}\text { Technical } \\
1.5\end{array}$ & 1.5 & $70-89$ & 112 & $76.25 \pm 18.04$ & $72.05 \pm 12.61$ & $0.98 \pm 0.27$ & $11.80 \pm 1.71$ & $116.25 \pm 3.59$ & $162.75 \pm 9.07$ \\
\hline Technical 2 & 2 & $90-109$ & 154 & $90.82 \pm 12.59$ & $102.04 \pm 6.83$ & $1.15 \pm 0.20$ & $12.63 \pm 1.11$ & $123.00 \pm 6.63$ & $169.88 \pm 9.14$ \\
\hline $\begin{array}{c}\text { Technical } \\
2.5\end{array}$ & 2.5 & $110-129$ & 98 & $99.50 \pm 13.09$ & $112.12 \pm 8.99$ & $1.15 \pm 0.20$ & $12.87 \pm 0.35$ & $127.00 \pm 7.35$ & $175.75 \pm 7.27$ \\
\hline Technical 3 & 3 & $130-149$ & 70 & $115.74 \pm 15.26$ & $139.35 \pm 7.82$ & $1.23 \pm 0.22$ & $13.00 \pm 0.00$ & $134.80 \pm 7.76$ & $168.20 \pm 10.18$ \\
\hline $\begin{array}{l}\text { Technical } \\
3.5\end{array}$ & 3.5 & $150-169$ & 84 & $105.92 \pm 11.83$ & $157.43 \pm 6.03$ & $1.50 \pm 0.16$ & $13.50 \pm 0.06$ & $145.83 \pm 5.85$ & $173.67 \pm 7.61$ \\
\hline Technical 4 & 4 & $>170$ & 84 & $104.08 \pm 2.24$ & $186.24 \pm 3.08$ & $1.79 \pm 0.01$ & $13.72 \pm 0.70$ & $155.00 \pm 1.41$ & $185.00 \pm 1.41$ \\
\hline \multicolumn{6}{|c|}{ Pearson's product moment correlation with Equivalent Training Load (r): } & 0.96 & 0.87 & 0.99 & 0.91 \\
\hline
\end{tabular}

Session-RPE showed an excellent correlation $(r=0.92)$ with the average heart rate recorded during the whole training session. This result is in concordance with a particular research study that has shown that session-RPE is related to the percentage of heart rate reserve during 30 min of steady-state running, as well as to the time duration at different intensities corresponding to heart rate at lactate thresholds (2.5 and $4.0 \mathrm{mmol} \cdot \mathrm{L}-1)$ during continuous and interval running [4]. Another study has also proved session-RPE to be significantly correlated to the heart rate-based method of quantifying internal training load proposed by Edwards [14] for endurance athletes [9]. More important for our study are the findings by Foster et al. [10], Impellizzeri et al. [5], and Manzi et al. [16] in college basketball players, young soccer players, and professional basketball players, respectively. Foster et al. [10] observed a consistent relationship in a collegiate men's basketball team between the session-RPE method and the heart rate method of monitoring the training. Impellizzeri et al. [5] monitored 19 young soccer players during a 7-week period. The training loads completed during that period were determined by multiplying the session-RPE (CR10-scale) by session duration in minutes. These session-RPE values were correlated to the training load measures obtained from three different heart ratebased methods suggested by Banister et al. [16], Edwards [14], and Lucia et al. [25]. All individual correlations between the various heart rate-based training loads and session-RPE were statistically significant ( $\mathrm{r}$ values from 0.50 to 0.85 ). Therefore, Impellizzeri et al. [5] concluded that session-RPE can be considered as a good indicator of global internal load in soccer training. Finally, Manzi et al. [16] also found significant relationships between individual session-RPE and all individual heart basemethods ( $\mathrm{r}$ values from 0.69 to 0.85 ) in 8 professional basketball players. Consequently, they demostrated that session-RPE may be considered as a viable method to assess training load without the use of more sophisticated tools (i.e. heart rate monitors), and most importantly, the session-RPE method even enabled the detection of periodisation patterns in the weekly planning for elite professional basketball players. The results in the present study are in the same line as those found in previous studies in team sports $[5,10,15,16,21]$ and showed that session-RPE may be considered as a valid method to assess training load in basketball. However, this finding was not the main goal of this study, for although both session-RPE and heart rate have been proved to be good indicators to control and monitor the training load, the main handicap still exists. They do not allow to periodise the training load before the practice, and the training load is not provided until the session has finished.

Most importantly, the variables obtained from the BATLOC tool (intensity, training load, and equivalent training load) had high correlation values with the sessionRPE $(r=0.90 ; r=0.80 ; r=0.76$, respectively) and the average heart rate $(r=0.69 ; r=0.87 ; r=0.78$, respectively) in the 700 individual training sessions (Table 2). These high correlations, obtained with methods (session-RPE and heart rate) that have been proved to be adequate to control and monitor training load in team sports [5,10,16], allow to confirm that the BATLOC tool may be a good instrument to measure training load in basketball. In the same way as the Borg scale (RPE) is considered to be a global indicator of exercise intensity, for it includes both physiological (oxygen uptake, heart rate, ventilation, beta endorphin, circulating glucose concentration, and glycogen depletion) and psychological factors [26], the BATLOC tool also covers the training load components (volume, intensity, density, and complexity) and the training load dimensions (cognitive, metabolic, and neuromuscular) proposed by Refoyo [27].

Finally, as expected, the correlations obtained between the BATLOC tool's variables (intensity, training load, and equivalent training load) and the maximum heart rate were not as high as the correlations found with session-RPE and heart rate, and they were even low. This finding can be explained and supported as follows: the training load obtained from the BATLOC tool, the session-RPE and the heart rate represent a single global rating of the intensity for the entire training session [10], while the maximum heart rate may show just a high-intensity moment or bout. Therefore, the maximum heart rate cannot be considered as an indicator of the total training load in a session.

To sum up the validation, the variables obtained from the BATLOC tool (intensity, training load and equivalent training load) were correlated to the training load previously calculated with the Foster et al. $[9,19]$ method (training load $=$ session-RPE $\mathrm{x}$ session duration in minutes). The values obtained were $r=0.83 ; r=0.97 ; r=0.96$, 
respectively. These correlations between the BATLOC tool and a method already validated and contrasted scientifically in basketball (i.e. the Foster et al. [9,10] method) show the validity of the BATLOC tool to control and monitor training load in basketball players.

Now that all the previous correlations have proved that the BATLOC tool may be a useful method to control and monitor the training load in basketball, the next step would be to validate the 8 different types of sessions established by the training load range (Tactical/Shot 0.5 , Technical 1 or pre-game session, Technical 1.5, Technical 2, Technical 2.5, Technical 3, Technical 3.5, and Technical 4). Basically, and in the same line as the 6-20 Borg scale is a range of numbers and verbal anchords that corresponds roughly to a heart rate range of $60 \mathrm{bpm}$ for number 6 to $200 \mathrm{bpm}$ for a score of 20 in healthy people (approximately 30 years of age) [23], one of the purposes of this study was to investigate if the type of sessions established could correspond to a session-RPE value and an average heart rate. For this purpose, average intensity, session-RPE, average heart rate, and maximum heart rate were correlated with the equivalent training load. The results obtained showed strong correlations $(\mathrm{r}=0.96$; $r=0.88 ; r=0.99$; $r=0.91$, respectively) (Table 3). Therefore, the value of RPE, the average heart rate, and the maximum heart rate related to any type of session may be established (i.e. Technical 1.5 session corresponds to a total training load of 70-89, a session-RPE of 11.80, and a mean heart rate of $116.25 \mathrm{bpm}$ ).

The previous data analysis and correlations obtained in this study suggest that the BATLOC tool is easy to use, quite reliable, and consistent with subjective (RPE) and objective physiological (heart rate) indices of the intensity of exercise training, which provides enough support to use it as a method of controlling and monitoring training load in basketball practices in real time. The BATLOC tool may offer a mechanism for quantifying the exercise intensity component and allows calculation of a single number representative of the combined intensity and duration of the training sessions while the practice is occurring.

In addition, the training load value calculated with the BATLOC tool showed high correlations with the body composition aspects in a professional first division male basketball team. The \% body fat decreased and the muscular mass increased as the training load increased. However, in periods when the training load was lower, the \% body fat increased and the muscular mass decreased (28).

Due to the fact that the BATLOC tool has been developed with the Excel software programme (Microsoft Corporation, U.S.), a daily exercise score is created. An exercise diary will show the daily and overall weekly training load, the latter being presented graphically, allowing the coach to have a visual impression of the periodisation plan. Finally, the originally planned periodisation with the daily and weekly training load is compared with the real daily and weekly load achieved.

To sum up we would like to highlight one of the most important limitations of the BATLOC tool: the session's training load calculated by the tool is for the team, not for individual players. Although, that value may be used to analysed and record the individual players' training load, it will not differentiate between players. For example, a team have players with different roles, physical characteristics and playing positions that influence the training load, however the tool will give you same value for each player.

\section{Conclusions}

To conclude, based on the results in this study and the literature reviewed, the BATLOC tool seems to be a good method to control global internal training load in basketball. This method does not require any expensive equipment and may be very useful and convenient for coaches to monitor the internal training load of basketball players. Furthermore, the present results suggest that the BATLOC tool may assist in the development of specific periodisation strategies for basketball teams. Finally, the BATLOC tool offers real-time feedback to basketball coaches, so that they can monitor the training load evolution during the training session and be able to modify the session exercises or tasks with the aim to achieve the required or planned training load.

\section{Acknowledgement}

We would like to express our gratitude to Spanish translator and interpreter Andrea Pérez-Arduña for the translation into English and style correction of the present paper.

\section{References}

[1] Coutts, A.J. \& Aoki, M.S. "Monitoring training in team sports". Olympic Laboratory: Technical Scientific Bulletin of the Brazilian Olympic Committe 2009; 9(2), 1-3.

[2] Gamble, P. "Periodization of training for team sports athletes". Strength and Conditioning Journal 2006; 28(5), 56-66.

[3] Viru, A. \& Viru, M. "Nature of training effects". In: Exercise and Sport Science, W. Garret and D. Kirkendall (Eds.). Philadelphia: Lippincott Williams \& Williams, 2000, 67-95.

[4] Foster, C., Hector, L.L., Welsh, R., Schrager, M., Green, M.A., \& Snyder, A.C. "Effects of specific versus cross-training on running performance". European Journal of Applied Physiology and Occupational Physiology 1995; 70(4), 367-372.

[5] Impellizzeri, F.M., Rampinini, E., Coutts, A.J., Sassi, A. \& Macora, S.M. "Use of RPE-based training load in soccer". Medicine \& Science in Sports \& Exercise 2004; 36(6), 1042-1047.

[6] Banister, E.W., Good, P., Holman, G. \& Hamilton, C.L. "Modelling the training response in athletes". In: Sport and Elite Performers. Laders MD ed. Champaign, IL: Human Kinetics, 1986; 7-23.

[7] Costill, D.L., Thomas, R., Robergs, R.A., Pascoe, D., Lambert, C., Barr, S., et al. "Adaptations to swimming training: influence of training volume”. Medicine and Science in Sports and Exercise 1991; 23(3), 371-377.

[8] Zatsiorsky, V. "Intensity of strength training facts and theory: Russian and Eastern European approach”. National Strength and Conditioning Association Journal 1992; 14(5): 46-57.

[9] Foster, C. "Monitoring training in athletes with reference to overtraining syndrome”. Medicine and Science in Sports and Exercise 1998; 30(7), 1164-1168.

[10] Foster, C., Florhaug, J.A., Franklin, J., Gottschall, L., Hrovatin, L.A., Parker, S., et al. "A new approach to monitoring exercise training”. Journal of Strength and Conditioning Research 2001; 15(1), 109-115.

[11] Gilman, M.B. "The use of heart rate to monitor the intensity of endurance training”. Sports Medicine 1996; 21, 73-79.

[12] Achten, J. \& Jeukendrup, A.E. "Heart rate monitoring: applications and limitations”. Sports Medicine 2003; 33, 517-538. 
[13] Morton, R.H., Fitz-Clarke, J.R. \& Banister, E.W. "Modeling human performance in running”. Journal of Applied Physiology 1990; 69(3), 1171-1177.

[14] Edwards, S. "High performance training and racing”. In: The heart rate monitor book, S. Edwards (Ed.). Sacramento, CA: Feet Fleet Press, 1993; 113-123.

[15] Coutts, A.J., Reaburn, P.R.J., Murphy, A.J., Pine, M.J. \& Impellizzeri, F.M. "Validity of the session-RPE method for determining training load in team sport athlete". Journal of Science and Medicine in Sport 2003; 6, 525.

[16] Manzi. V., D’Ottavio, S., Impellizzeri, F.M., Chaouachi, A., Chamri, K. \& Castagna, C. "Profile of weekly training load in elite male professional basketball players". Journal of Strength and Conditioning Research 2010; 24(5), 1399-1406.

[17] Moreira. A, de Freitas, C.G., Nakamura, F.Y., Aoki, M.S "Session RPE and stress tolerante in young volleyball and basketball players". Brazilian Journal of Kinantropometry and Human Performance 2010; 12(5), 345-352.

[18] Berdejo-del-Fresno, D. \& González-Ravé, J.M. "Development of a new method to monitor and control the training load in basketball: the BATLOC Tool”. Journal of Sport and Health Research 2012; 4(1), 93-102.

[19] Anderson, L., Triplett-McBride, T., Foster, C., Doberstein, S. \& Brice, G. "Impact of training patterns on incidence of illness and injury during a women's collegiate basketball season”. Journal of Strength and Conditioning Research 2003; 17(4), 734-738.

[20] Stagno, K.M., Thatcher, R. \& Van Someren, K.A. "A modified TRIMP to quantify the in-season training load of team sport players”. Journal of Sports Science 2007; 25(6), 629-634.
[21] Coutts, A.J., Rampinini, E., Marcora, S.M., Castagna, C. \& Impellizzeri, F.M. "Heart rate and blood lactate correlates of perceived exertion during small-sided soccer games". Journal of Science and Medicine in Sport 2009; 12, 79-84.

[22] Lohmann, T.G., Roche, A.F. \& Martorell, R. Anthropometric standardization reference manual. Champaign, IL: Human Kinetics. 1988.

[23] Borg, G. "Perceived exertion as an indicator of somatic stress". Scandinavian Journal of Rehabilitation Medicine 1970; 2, No. 2-3, 92-98.

[24] Fleiss, J.L. The design and analysis of clinical experiments. New York, NY: Wiley. 1986

[25] Lucía, A., Hoyos, J., Carvajal, A. \& Chicharro, J.L. "Heart rate response to professional road cycling: The Tour de France". International Journal of Sports Medicine 1999; 20, 167-172.

[26] Morgan, W.P. "Psychological factors influencing perceived exertion”. Medicine and Science in Sports and Exercise 1994; 26, 1071-1077.

[27] Refoyo, I. La decisión táctica de juego y su relación con la respuesta biológica de los jugadores: una aplicación al baloncesto como deporte de equipo. PhD Thesis. Universidad Complutense de Madrid. 2001.

[28] Berdejo-del-Fresno, D., Sánchez-Pérez, S. \& Jiménez-Díaz, J.F. "Body composition and training load in basketball: a direct connection in the high level”. EFDeportes. Revista Digital. 2008; Year 13, N 119. 